\title{
Respiration Rate, Growth Rate and the Accumulation of Streptomycin in Escherichia coli
}

\author{
By MARIANNE E. MUIR, MERCEDES BALLESTEROS AND \\ BRIAN J. WALLACE* \\ School of Microbiology, Faculty of Biological Sciences, The University of New South Wales, \\ Kensington, NSW 2033, Australia
}

(Received 23 January 1985; revised 30 April 1985)

\begin{abstract}
Using chemostat cultures of Escherichia coli it was possible to vary respiration rates while maintaining a constant growth rate. This allowed the effect of variations in respiration rates on the accumulation of streptomycin to be studied in cultures at constant growth rates. At a particular dilution rate cultures exhibited higher respiration rates when phosphate limited growth than when carbon limited growth. A ubiquinone-deficient strain had a lower rate of respiration at a particular dilution rate than a related ubiquinone-sufficient strain. In spite of these differences in respiratory activity, the accumulation of streptomycin was identical in carbon- and in phosphate-limited chemostat cultures of ubiquinone-deficient and ubiquinonesufficient strains. Moreover, accumulation of streptomycin in an anaerobic chemostat culture occurred at the same rate as that in an aerobic chemostat. There was however a lag of $1.5 \mathrm{~h}$ before accumulation commenced in the anaerobic culture, a feature that was not apparent in the aerobic culture. These results indicate that the lower rates of respiration in slow-growing bacteria are not responsible for the decreased accumulation of streptomycin in slow-growing compared to fast-growing cultures. Moreover, it seems unlikely that quinones are involved directly (e.g. as carriers) in streptomycin accumulation, since removal of $90 \%$ of cellular ubiquinone, or replacement of ubiquinone with a structural analogue, did not affect accumulation as long as mutant and parent cultures grew at the same rate.
\end{abstract}

\section{INTRODUCTION}

In a previous study using chemostat cultures of Escherichia coli and Bacillus megaterium, the rate of accumulation of streptomycin was proportional to the growth rate of the culture before addition of the antibiotic (Muir et al., 1984). That is, streptomycin was accumulated more rapidly by fast-growing than by slow-growing bacteria. Respiration rates of bacteria also vary with dilution rates. Thus for chemostat cultures of Klebsiella aerogenes, where growth was limited by available carbon source, respiration rates were greater for cultures at higher than at lower dilution rates (Neijssel \& Tempest, 1975). In view of recent studies (see Discussion), which point to the importance of respiratory energy in streptomycin accumulation, we considered it was possible that the rapid respiration rates of fast-growing cultures were responsible for the greater accumulation of streptomycin observed at higher dilution rates. We therefore investigated the relationship between growth rates, respiration rates and the rates of accumulation of streptomycin in chemostat cultures of $E$. coli.

Abbreviations: AUM, aminoglycoside uptake medium; CCCP, carbonyl cyanide $m$-chlorophenylhydrazone. 


\section{METHODS}

Bacterial strains. All strains used were derivatives of E. coli K12. Strain JP2140 [ilv-I his-29(am) trpA9605(am)] was obtained from A. J. Pittard, University of Melbourne, Australia. The streptomycin-sensitive strains NSW60 (ubiA420 menA40l) and NSW62 (menA40I) were constructed in the following way. Strain AN144 (Hfr ubiA420 metB rpsL rel), obtained from I. G. Young, Australian National University, Canberra, Australia, was mated overnight in L-broth with NSW33, a spontaneous nalidixic acid-resistant derivative of JP2140. A recombinant clone, NSW56 (metB ubiA420), unable to grow on succinate as sole source of carbon, was isolated. Strain NSW60 (ubiA420 menA40I) is a $\mathrm{met}^{+}$transductant derived from a cross using NSW56 and phage $\mathrm{P} l_{\mathrm{kc}}$ propagated on a menA401 strain (AN386). Strain NSW62 (menA401) was derived by selecting a clone capable of growth on succinate following a transduction cross with NSW60 and phage $\mathrm{Pl}_{\mathrm{kc}}$ from a $u b i^{+}$strain. Genetic nomenclature conforms to that recommended by Demerec et al. (1968), and the symbols for gene loci are those of Bachmann \& Low (1980).

Media and growth of cells. Cultures were maintained on plates consisting of nutrient broth (Oxoid) enriched with $0.5 \%(\mathrm{w} / \mathrm{v})$ brain heart infusion (Oxoid) and supplemented with $2 \%(\mathrm{w} / \mathrm{v})$ agar (Difco). Cultures from transduction and conjugation experiments were grown on plates consisting of medium A (Davis \& Mingioli, 1950) with $2 \%$ agar, supplemented with glucose or succinate (each $30 \mathrm{~mm})$, thiamin. $\mathrm{HCl}(3 \mu \mathrm{M}), \mathrm{L}$-isoleucine $(0 \cdot 38 \mathrm{mM}), \mathrm{L}$-valine $(0.43 \mathrm{mM})$, L-histidine $(0.52 \mathrm{mM})$, L-tryptophan $(0.2 \mathrm{mM})$, L-methionine $(0.17 \mathrm{~mm})$, p-hydroxybenzoate $(0.1$ or $1.0 \mathrm{~mm})$, uracil $(0.2 \mathrm{~mm})$ and nalidixic acid $\left(50 \mu \mathrm{g} \mathrm{m}^{-1}\right)$, as required. For all batch culture experiments, strains were grown in nutrient broth supplemented with $0.5 \%(\mathrm{w} / \mathrm{v})$ brain heart infusion. Generation times (doubling times) for batch cultures were calculated from the slope of the linear (exponential) portions of plots of log OD 600 against time. For growth in continuous culture, aminoglycoside uptake medium (AUM; Bryan \& Van Den Elzen, 1977) was used as previously described (Muir et al., 1984). Growth was limited by availability of either carbon [1, 2 or $5 \mathrm{~mm}$-glucose plus $0 \cdot 1 \%(\mathrm{w} / \mathrm{v})$ Casamino acids (Difco)] or $\mathrm{K}_{2} \mathrm{HPO}_{4}(0 \cdot 1 \mathrm{~mm}$ ) since increasing the concentration of either glucose (or Casamino acids) or $\mathrm{K}_{2} \mathrm{HPO}_{4}$, in the carbon-limited and phosphate-limited chemostats respectively, resulted in higher cell densities. The $\mathrm{pH}$ of chemostat cultures in steady-state at the dilution rates used was the same as that of the uninoculated medium.

For anaerobic growth, the complete chemostat apparatus including a flask containing $400 \mathrm{ml}$ sterile AUM was autoclaved at $0.069 \mathrm{MPa}$ for $25 \mathrm{~min}$ in order to dispel oxygen from the medium and sterilize the apparatus. When the medium had cooled to about $50^{\circ} \mathrm{C}$, nutrient solutions were added aseptically. The chemostat was then inoculated with $3 \mathrm{ml}$ culture, and the apparatus assembled and flushed with nitrogen (instrument grade; CIG, Australia) for several hours. After equilibration of culture and medium had occurred in the oxygen-free environment, medium flow to the chemostat was commenced. Nitrogen was bubbled continuously through the sparger to mix cells and maintain the anaerobic environment. Syringes for sample withdrawal were flushed with nitrogen before and after use. The absence of oxygen in the chemostat was confirmed using a Clark-type oxygen electrode. The chemostat apparatus and medium for the aerobic (control) experiment were prepared in an identical manner except that air was supplied instead of nitrogen and medium flow was initiated as soon as the chemostat was inoculated. Growth was monitored by OD measurements in a Spectronic 20 spectrophotometer at $600 \mathrm{~nm}$ or in a Klett-Summerson colorimeter fitted with a blue filter.

Streptomycin accumulation experiments. Streptomycin was mixed with $\left[{ }^{3} \mathrm{H}\right]$ dihydrostreptomycin (Amersham) to give a specific activity of 169 d.p.m. (ng streptomycin) ${ }^{-1}$. The final concentration of streptomycin in all experiments was $20 \mu \mathrm{g} \mathrm{ml}^{-1}$. Accumulation experiments in batch and continuous cultures were done as previously described (Muir et al., 1984).

Measurement of respiration rates in chemostat cultures. Respiration rates were measured by trapping and counting the ${ }^{14} \mathrm{CO}_{2}$ that was evolved from [ $\left.{ }^{14} \mathrm{C}\right]$ glucose. The concentration of glucose in the medium was $10 \mathrm{mM}(0 \cdot 1 \mu \mathrm{Ci}$; $3.7 \mathrm{kBq}) \mathrm{ml}^{-1}$ and $2 \mathrm{mM}(0.02 \mu \mathrm{Ci} ; 0.74 \mathrm{kBq}) \mathrm{ml}^{-1}$ for phosphate- and carbon-limited cultures respectively. Gases from the chemostat vessel passed through an acid reservoir to the $\mathrm{CO}_{2}$ trapping system which consisted of a sealed scintillation vial containing ethanolamine $(2 \mathrm{ml})$ and methanol $(4 \mathrm{ml})$. The seal on the scintillation vial was penetrated by a narrow necked glass tube which allowed bubbling of gases through a liquid column of ethanolamine and methanol. The $\mathrm{CO}_{2}$ was collected for $1 \mathrm{~h}$ after which time the glass tube was washed twice with methanol $(2 \mathrm{ml})$ into the scintillation vial. A fresh vial and trap was connected at hourly intervals and collection continued until a constant evolution rate of $\mathrm{CO}_{2}$ was established (usually five or six samples). Aquassure scintillation fluid ( $5 \mathrm{ml}$; New England Nuclear) was added and the vials were counted in a Packard Tricarb liquid scintillation spectrometer (model 300). The five or six values for d.p.m. were averaged for calculation of respiration rates which are expressed as d.p.m. in $\mathrm{CO}_{2}$ evolved $\mathrm{h}^{-1}$ (mg dry wt bacteria) $)^{-1}$.

The efficiency of the $\mathrm{CO}_{2}$ trapping system was tested by adding $\mathrm{NaH}^{14} \mathrm{CO}_{3}$ to the chemostat vessel which contained $1 \mathrm{M}-\mathrm{HCl}: 90 \%$ of the radioactive biocarbonate was recovered as ${ }^{1+} \mathrm{CO}_{2}$.

Estimation of quinones in whole cells. Bacteria grown in batch cultures of nutrient broth supplemented with $0.5 \%$ $(w / v)$ brain heart infusion were extracted with acetone and the concentration of quinones, was determined by thinlayer chromatography on silica gel G plates as described previously (Muir et al., 1981). 


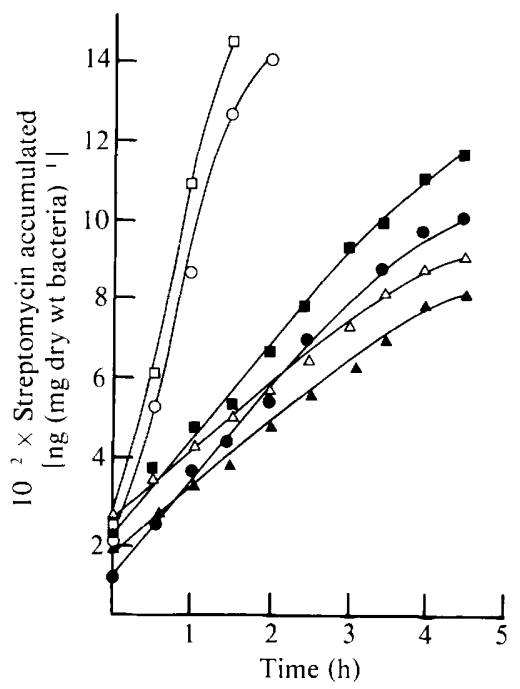

Fig. 1. Streptomycin accumulation in chemostat cultures of E. coli. strains JP2140, NSW60 (uhiA menA) and NSW62 (ubi+ menA) under either carbon or phosphate limitation. Strain JP2140 was grown in chemostats in AUM with limiting phosphate at $D=0.25 \mathrm{~h}^{-1}(O)$ and $D=0.6 \mathrm{~h}^{-1}(O)$, or

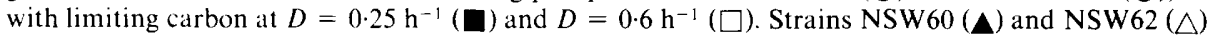
were grown similarly under phosphate limitation and at $D=0.25 \mathrm{~h}^{-1}$. The medium for strain NSW60 contained $p$-hydroxybenzoate $(0.1 \mathrm{mM})$. When steady-state growth was reached streptomycin $\left(20 \mu \mathrm{g} \mathrm{ml}^{-1}\right)$ was added to the culture vessel and medium reservoir and samples were taken and measured for accumulation of streptomycin (see Methods).

Table 1. Respiration rates in carbon-or phosphate-limited chemostats of E. coli strains JP2140, NSW60 and NSW62

Strains JP2140, NSW60 (ubiA menA) and NSW62 (ubi+ menA) were grown in chemostats at the dilution rates $(D)$ indicated under either carbon or phosphate limitation. Rates of evolution of $\mathrm{CO}_{2}$ were measured as described in Methods. Results are the mean of three experiments and are expressed as d.p.m. in $\mathrm{CO}_{2}$ evolved $\mathrm{h}^{-1}$ (mg dry wt bacteria) $)^{-1}$.

$\begin{array}{lllc}\text { Strain } & \begin{array}{c}D \\ \left(\mathrm{~h}^{-1}\right)\end{array} & \overbrace{\text { Phosphate-limited }}^{\text {Cespiration rate }} \\ \text { JP2140 } & 0.25 & 1.78 \pm 0.12 \times 10^{6} & 6.66 \pm 0.12 \times 10^{5} \\ \text { JP2140 } & 0.6 & 1.95 \pm 0.10 \times 10^{6} & 1.22 \pm 0.03 \times 10^{6} \\ \text { NSW60* } & 0.25 & 6.52 \pm 0.52 \times 10^{5} & \text { NT } \\ \text { NSW62 } & 0.25 & 1.12 \pm 0.14 \times 10^{6} & \text { NT }\end{array}$

NT, Not tested.

* Medium contained $p$-hydroxybenzoate $(0 \cdot 1 \mathrm{~mm})$.

\section{RESULTS}

Respiration rates and accumulation of streptomycin in carbon-limited and phosphate-limited chemostat cultures

As expected from previous results (Neijssel \& Tempest, 1975), respiration rate, as measured by evolution of $\mathrm{CO}_{2}$, increased with increasing dilution rate in carbon-limited chemostat cultures of E. coli strain JP2140 (Table 1). Accumulation of streptomycin was also greater in faster growing cultures (Fig. 1; Muir et al., 1984). At a lower dilution rate $\left(D=0.25 \mathrm{~h}^{-1}\right), \mathrm{CO}_{2}$ evolution in a phosphate-limited chemostat culture was approximately three times greater than that in a carbon-limited culture (Table 1). This result is consistent with that of Neijssel \& Tempest (1976). Though the respiration rate in the phosphate-limited culture was almost threefold greater than in the carbon-limited culture, the rates of accumulation of streptomycin were the same for both (Fig. 1). 


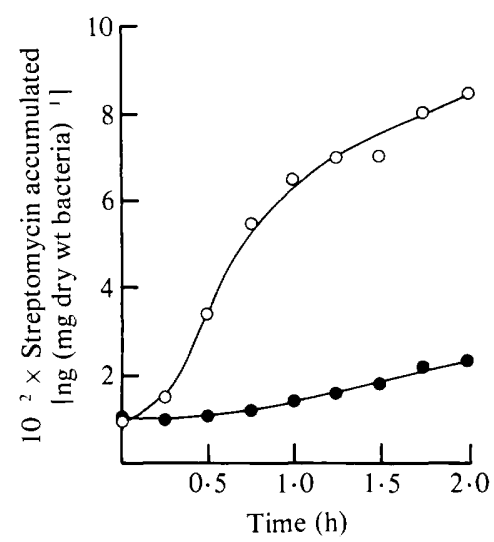

Fig. 2

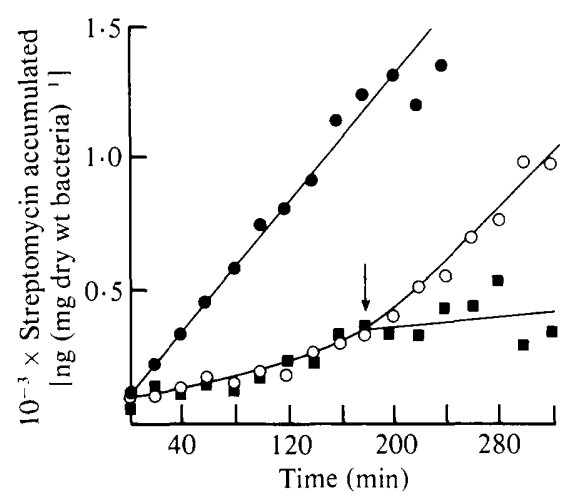

Fig. 3

Fig. 2. Streptomycin accumulation in batch cultures of strains NSW60 (ubiA menA) (O) and NSW62 $(m e n A)(O)$. Strains were grown to early exponential phase in nutrient broth $(10 \mathrm{ml})$ supplemented with brain heart infusion $(0.5 \%, \mathrm{w} / \mathrm{v})$. The medium for strain NSW60 was supplemented with $p$ hydroxybenzoate $(0.1 \mathrm{mM})$. Streptomycin $\left(20 \mu \mathrm{g} \mathrm{ml}^{-1}\right)$ was added and samples were removed at intervals for determination of streptomycin accumulation (see Methods).

Fig. 3. Streptomycin accumulation in chemostat cultures under aerobic and anaerobic conditions. Strain NSW62 (menA) was grown in a chemostat in AUM (modified by omitting nitrate) and supplemented with glucose ( $1 \mathrm{~mm}$ for aerobic cultures, $5 \mathrm{~mm}$ for anaerobic cultures). Casamino acids $(0.1 \%, \mathrm{w} / \mathrm{v}), \mathrm{KHCO}_{3}(20 \mathrm{~mm})$ and uracil $(0.2 \mathrm{~mm})$ were also present. The anaerobic chemostat was continuously flushed with nitrogen. Dilution rates for aerobic and anaerobic experiments were identical $\left(D=0 \cdot 34 \mathrm{~h}^{-1}\right)$. When steady-state growth was reached streptomycin was added and accumulation was measured (see Methods). Accumulation under aerobic conditions ( $)$ ); accumulation under anaerobic conditions with CCCP $(10 \mu \mathrm{M})$ added $(\mathbf{a})$ at the time indicated by the arrow or without CCCP addition (O).

\section{Respiration rates and accumulation of streptomycin in ubiA menA strains of E. coli}

To study the effects of a decrease in cellular ubiquinone on respiration and on accumulation of streptomycin, we measured the rates of evolution of $\mathrm{CO}_{2}$ and of streptomycin accumulation in a ubiquinone-deficient strain of $E$. coli (NSW60;ubiA men $A$ ) and its parent (NSW62;ubi+ men $A$ ). We chose the men $A$ strain so that menaquinone and demethylmenaquinone, which would have been present in $\mathrm{men}^{+}$strains, could not influence the results. It had previously been found (M. Muir, unpublished results) that removal of menaquinone and demethylmenaquinone had no effect on aerobic respiration and only a slight negative effect on accumulation of streptomycin. In phosphate-limited chemostat cultures of strain NSW62 $\left(u b i^{+} m e n A\right)$ the respiration rate was 1.7 times that of strain NSW60 (ubiA menA) at $D=0.25 \mathrm{~h}^{-1}$ (Table 1) whereas the rates of accumulation of streptomycin in the two strains were identical (Fig. 1). In batch culture, however, accumulation of streptomycin was much greater for strain NSW62 than for strain NSW60 (Fig. 2). Generation times for batch cultures of strains NSW60 and NSW62 were $130 \mathrm{~min}$ and $25 \mathrm{~min}$ respectively.

Both batch and chemostat cultures of strain NSW60 were supplemented with $p$ hydroxybenzoate $(0 \cdot 1 \mathrm{mM})$ to allow the production of some ubiquinone (Wallace \& Young, 1977). When harvested from batch cultures, strains NSW60 and NSW62 contained 26 and $226 \mathrm{ng}$ ubiquinone (mg dry wt bacteria) ${ }^{-1}$ respectively. No menaquinone or demethylmenaquinone could be detected in either strain.

\section{Accumulation of steptomycin in an anaerobic chemostat culture of E. coli}

The finding that a ubiquinone-deficient strain with a lower respiration rate accumulated streptomycin at the same rate as a $u b i^{+}$strain provided that growth rates were taken into account (Fig. 1) prompted us to consider the possibility that the lowered accumulation of aminoglycosides by facultative anaerobes growing anaerobically might be accounted for (at least partially) by slower growth rates. For example, a batch culture of strain JP2140 growing 
aerobically in AUM with glucose and Casamino acids had a generation time of 36 min whereas anaerobically its generation time was $72 \mathrm{~min}$. To avoid effects that differences in growth rates might have, we measured the accumulation of streptomycin in chemostat cultures growing aerobically and anaerobically at the same dilution rate. To eliminate the menaquinonedependent electron transport to fumarate that may occur under anaerobic conditions (Kroger, 1978), strain NSW62 (menA) was used for these experiments. AUM was modified by omitting nitrate $\left(\mathrm{NH}_{4} \mathrm{Cl}\right.$ replaced $\left.\mathrm{NH}_{4} \mathrm{NO}_{3}\right)$ which, if present, might act as an electron acceptor. Uracil $(0.2 \mathrm{mM})$ and $\mathrm{K} \mathrm{HCO}_{3}(20 \mathrm{~mm})$ were also added to aerobic and anaerobic cultures. In addition to Casamino acids $(0.1 \%, \mathrm{w} / \mathrm{v})$, the anaerobic culture contained glucose $(5 \mathrm{mM})$ (aerobic, $1 \mathrm{mM})$ in order to provide similar cell densities at steady-state in both cultures. The $\mathrm{pH}$ of aerobic and anaerobic cultures was $7 \cdot 0$, indicating that no $\mathrm{pH}$ decrease was associated with anaerobic growth at the glucose concentrations used.

Accumulation of streptomycin occurred at similar rates in aerobic and anaerobic chemostat cultures of strain NSW62 ( $D=0.34 \mathrm{~h}^{-1}$; Fig. 3). However, accumulation in the anaerobic culture was preceded by a $1.5 \mathrm{~h}$ lag, whereas no lag was evident for the aerobic culture. Accumulation of streptomycin under anaerobic conditions was abolished by carbonyl cyanide $m$-chlorophenylhydrazone (CCCP; $10 \mu \mathrm{M})$ added $180 \mathrm{~min}$ after the streptomycin.

\section{DISCUSSION}

In this study we dissociated growth rates from respiration rates by three means. The rate of respiration was higher in chemostat cultures at a fixed dilution rate when phosphate rather than carbon was the growth-limiting nutrient. Decreases in respiration rates were accomplished using chemostat cultures of a respiratory mutant and by growth of a non-respiratory mutant under anaerobic conditions. In all cases the rate of accumulation of streptomycin remained unaffected by changes in respiratory activity. It must be noted however that accumulation under anaerobic conditions was preceded by a lag that was not apparent in the corresponding aerobic culture. This lag may explain the heightened resistance to streptomycin that is characteristic of facultative anaerobes under anaerobic conditions (Kogut et al., 1965).

Muir et al. (1981) reported the isolation and characterization of a respiratory mutant of $E$. coli K 12 (strain NSW77) with decreased accumulation of aminoglycosides in batch culture. This strain harboured a mutation at $u b i F$, a gene encoding the enzyme that catalyses the penultimate step of ubiquinone biosynthesis (Young et al., 1973). Another ubiquinone-deficient strain (ubiD) and a haem-deficient strain of $E$. coli also had decreased accumulation of aminoglycosides (Campbell \& Kadner, 1980; Bryan \& Van Den Elzen, 1977). Mutant strains of other genera of bacteria possessing both low level resistance to aminoglycosides and defective respiratory metabolism have been isolated. Thus, cytochrome-deficient strains of Pseudomonas aeruginosa (Bryan et al., 1980; Bryan \& Kwan, 1981), menaquinone-deficient strains of Bacillus subtilis (Taber et al., 1981: Taber \& Halfenger, 1976) and haem mutants of Staphylococcus aureus (Tien \& White, 1968; Kaplan \& Dye, 1976; Miller et al., 1980) were isolated from wild-type populations by selection for increased resistance to aminoglycoside antibiotics. These observations, in addition to those indicating that aminoglycoside uptake and kill are decreased in the presence of respiratory inhibitors such as azide and cyanide, and under anaerobic conditions, have led to the view that aminoglycoside accumulation is influenced by respiratory activity.

For the ubiA strain (NSW60; Fig. 1) and the ubiF strain (NSW77; M. Muir, unpublished results) the accumulation of streptomycin in chemostat cultures of mutant and parent was identical provided that dilution rates were the same. It seems possible that the previously observed decreases in aminoglycoside accumulation by various respiratory-deficient mutants, and by batch cultures of facultative anaerobes growing anaerobically, can be accounted for by differences in growth rates between parent and mutant strains, and between aerobic and anaerobic cultures. For example, the $u$ biD mutant with a decreased uptake of streptomycin in batch cultures (Bryan \& Van Den Elzen, 1977) has a generation time of $2.5 \mathrm{~h}$ compared with $1.25 \mathrm{~h}$ for the wild-type strain AB2154 (Newton et al., 1972). A twofold difference in growth rate 
between parent and mutant could explain the decreased uptake of streptomycin observed. With $B$. subtilis, which contains menaquinone but not ubiquinone, a menaquinone requirement for gentamicin uptake was manifest when the menaquinone content of cells was decreased to less than $10 \%$ of the normal value (Taber et al., 1981). It is probable that loss of more than $90 \%$ of the cellular menaquinone had a deleterious effect on growth rate which would serve to decrease accumulation of the aminoglycoside. Campbell \& Kadner (1980) found that uptake of dihydrostreptomycin could be partially restored in anaerobic batch cultures by the addition of nitrate. This restoration might result from a stimulation in growth rate due to an increase in electron transport activity.

It has been proposed that the bacterial quinones ubiquinone and menaquinone play a role in aminoglycoside accumulation as membrane carriers of the antibiotic in $E$. coli (Bryan \& Van Den Elzen, 1977) and B. subtilis (Taber et al., 1981) respectively. The results of this paper suggest that neither ubiquinone nor menaquinone are directly involved in streptomycin accumulation in $\dot{E}$. coli. Thus when the concentration of ubiquinone was substantially decreased in the $u b i A$ strain (and the menaquinone and demethylmenaquinone removed entirely), the respiration rate was decreased but no effect on streptomycin accumulation was observed. Complete replacement of ubiquinone in the $u b i F$ strain by a structural analogue, 2-octaprenyl-3-methyl-6-methoxy-1,4benzoquinone, though causing a decrease in respiration rate (Muir et al., 1981), did not affect the accumulation of streptomycin in chemostat cultures. This suggests either that ubiquinone is not involved in streptomycin accumulation, or that the analogue can take over the role of ubiquinone without loss of efficiency.

The authors wish to acknowledge the skilled technical assistance of Tillie Coster. We also thank I. G. Young for strains AN/44 and AN386 and A. J. Pittard for strain JP2140. This project was supported by a grant to B.J.W. from the Australian Research Grants Committee.

\section{REFERENCES}

BachmanN, B. J. \& Low, K. B. (1980). Linkage map of Escherichia coli $\mathrm{K}-12$; edition 6. Microbiological Revien's 44, 1-56.

Bryan, L. E. \& KWAN, S. (1981). Aminoglycosideresistant mutants of Pseudomonas aeruginosa deficient in cytochrome d, nitrate reductase and aerobic transport. Antimicrohial Agents and Chemotherapy 19, 958-964.

Bryan, L. E. \& Van Den Elzen, H. M. (1977). Effects of membrane-energy mutations and cations on streptomycin and gentamicin accumulation by bacteria: a model for entry of streptomycin and gentamicin in susceptible and resistant bacteria. Antimicrobial Agents and Chemotherapy 12, 163-177.

Bryan, L. F., Nicas, T., Holloway, B. W. \& Crowther, C. (1980). Aminoglycoside-resistant mutation of Pseudomona aeruginosa defective in cytochrome $\mathrm{C}_{552}$ and nitrate reductase. Antimicrobial Agents and Chemotherapy 17, 71-79.

CAMpbell, B. D. \& K ADNER, R. J. (1980). Relation of aerobiosis and ionic strength to the uptake of dihydrostreptomycin in Escherichia coli. Biochimica et biophysica acta 593, 1-10.

Davis, B. D. \& Mingioli, E. S. (1950). Mutants of Escherichia coli requiring methionine or vitamin B12. Journal of Bacteriolog! 60, 17-28.

Demerec, M., Adelberg, E. A., Clark, A. J. \& Hartman, P. E. (1968). A proposal for a uniform nomenclature in bacterial genetics. Journal of General Microhiology. 50, 1-14.

Kaplan, M. L. \& Dye, W. E. (1976). Growth requirement of some small-colony-forming variants of Staphylococcus aureus. Journal of Clinical Microbiology 4, 343-348.

Kogut, M., Lightbown, J. W. \& Isaacson, P. (1965). Streptomycin action and anaerobiosis. Journal of General Microbiology 39, 155-164.

KROGER, A. (1978). Fumarate as terminal acceptor of phosphorylative electron transport. Biochimica et biophysica acta 505, 129-145.

Miller, M. H., Edberg, S. C., Mandel, L. J., Behar, C. F. \& Steigbigel, N. H. (1980). Gentamicin uptake in wild-type and aminoglycoside-resistant small-colony mutants of Staphylococcus aureus. Antimicrobial Agents and Chemotherapy 18, 722-729.

Muir, M. E., Hanwell, D. R. \& Wallace, B. J.. (1981). Characterization of a respiratory mutant of Escherichia coli with reduced uptake of aminoglycoside antibiotics. Biochimica et biophysica acta $\mathbf{6 3 8}$, 234-241.

Muir, M. E., van Heeswyck, R. S. \& Wallace, B. J. (1984). Effect of growth rate on streptomycin accumulation by Escherichia coli and Bacillus megaterium. Journal of General Microbiology 130, 2015 2022.

Neijssel, O. M. \& Tempest, D. W. (1975). The regulation of carbohydrate metabolism in Klebsiella aerogenes NCTC 418 organisms growing in chemostat culture. Archives of Microbiology 106, 251-258.

Neijssel, O. M. \& Tempest, D. W. (1976). Bioenergetic aspects of aerobic growth of Klebsiella aerogenes NCTC 418 in carbon-limited and carbon-sufficient chemostat cultures. Archives of Microbiology 107, 215-221. 
Newton, N. A., Cox, G. B. \& Gibson, F. (1972). Function of ubiquinone in Escherichia coli: a mutant strain forming a low level of ubiquinone. Journal of Bacteriology 109, 69-73.

TABER, H. \& Halfenger, G. M. (1976). Multipleaminoglycoside-resistant mutants of Bacillus subtilis deficient in accumulation of kanamycin. Antimicrobial Agents and Chemotherapy 9, 251-259.

Taber, H. W., Sugarman, B. J. \& Halfenger, G. M (1981). Involvement of menaquinone in the active accumulation of aminoglycosides by Bacillus subtilis. Journal of General Microbiology 123, 143-149.

TiEn, W. \& White, D. C. (1968). Linear sequential arrangement of genes for the biosynthetic pathway of protoheme in Staphllococcus aureus. Proceedings of the National Academy of Sciences of the United States of America 61, 1392-1398.

Wallace, B. J. \& Young, I. G. (1977). Role of quinones in electron transport to oxygen and nitrate in Escherichia coli: studies with a ubiA, menA double quinone mutant. Biochimica et biophysica acta 461, $84-100$.

Young, I. G., Stroobant, P., Macdonald, C. G. \& Gibson, F. (1972). Pathway for ubiquinone biosynthesis in Escherichia coli $\mathrm{K}-12$ : gene-enzyme relationships and intermediates. Journal of Bacteriology 114, 42-52. 